\title{
Flexible Arm Optimal Design of Pipe Robot Expanding Brake Structure
}

\author{
Han $\mathrm{Ni}^{1}$, Yulian Chang, Sheng Gao \\ Northeast Petroleum University, Daqing, 163318 China \\ ${ }^{1}$ hljdqnh@163.com
}

Keywords: Flexible arm; nonlinear deflection; optimal design

\begin{abstract}
Structure parameters of Flexible arm in expanding brake of pipe robot are important, which are used to effect the expanding force. Pseudo-rigid-body model is often adopted for size optimization. The relationship between stress distortion and structure parameters are analyzed by virtual principle. The optimization objective is to make sure that piston has the least propulsive force without reducing expanding force. The mechanical model is established, which is used to calculate structural parameters of flexible arm in expanding brake for pipe robot. And results are illustrated by some experiments. A simple optimal design method is proposed for nonlinear elastic deflections, which has great value for centralizer and bow spring centralizer of pipe robot in Petroleum and petrochemical industry.
\end{abstract}

Pipe is one of the most important ways for mass and energy transfer, which is widely used in nuclear power, gas, and petroleum systems. There are also many problems of pipe detection and repairment. Some research centers pay high attention for developing pipe robot. Commonly, there are three parts of pipe robot including expanding brake, expansion joints, and centralizer, illustrated in Figure 1.

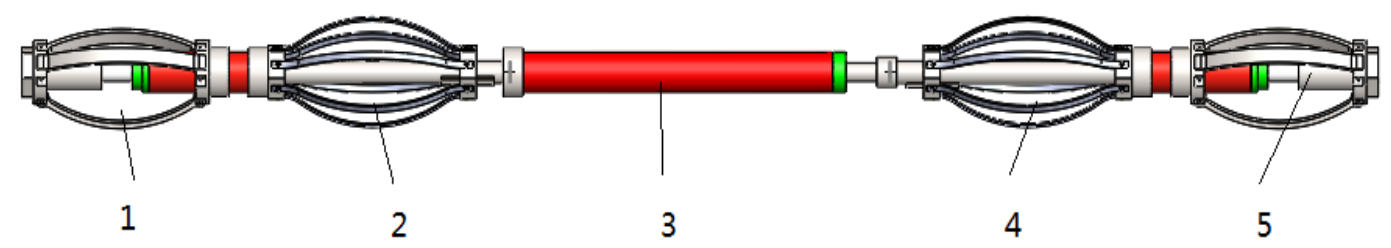

1, 5-expanding brake; 2, 4- centralizer; 3-expansion joints

Figure 1 Structure diagram of pipe robot

The function of expanding brake is to provide support for pipe robot while walking, and axial traction movement, which is the stressed member. The structure of expanding brake is sketched. Distending force depends on stiffness coefficient of flexible arm and trust of piston rod. Once trust of piston is determined, stiffness coefficient of flexible arm will be the important influencing factor. This means dimension parameters and their combination should be optimized. Generally, elliptic integrals and nonlinear finite element analysis are often adopted to solve these problems, both of which can be used in calculation test while choosing design scheme. They are seldom applied in optimization stage. Pseudo-rigid-body model is proposed in this paper to analyze relationship between forces, deflection and structure parameter, which can also be used to optimize structure parameters.

\section{Elastostatic analysis of flexible arm}

Bearing joint is chosen as the origin, and the coordinate system can be established, denoted by $x$ oy, illustrated as Figure 3. Point $\mathrm{O}$ is the fixed hinged support, and point $\mathrm{B}$ is the active hinged support, which can move along OB. The length of flexible arm is $l$, where the solid line denotes the initial status, and the dashed line denotes the deformed status under the force $F$. States parameters of flexible arm are denoted by $R_{0}, a_{0}, b_{0}, \theta_{0}$, and $R, a, b, \theta$, respectively, which represent initial status and deformed status. Inverse forces of bearing joint are listed as $F_{x 0}, F_{y 0}, F_{y B}$. 


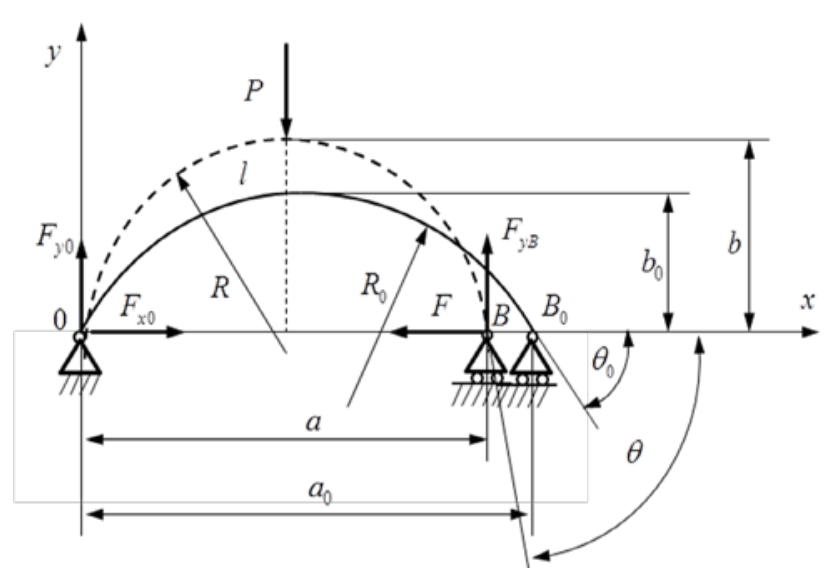

Figure 3 Strain deforming sketch of flexible arm

Based on the force balance condition, we can get $F_{x 0}=F, F_{y 0}=F_{y B}=P / 2$.

According to the Bernoulli-Euler equation $\frac{1}{R_{0}}=\frac{d \theta}{d s}$.Integrating above equation, we can get initial position of both horizontal and vertical direction of point $\mathrm{B}$ :

$$
\left\{\begin{array}{c}
a_{0}=2 R_{0} \sin \theta_{0} \\
b_{0}=R_{0}\left(1-\cos \theta_{0}\right)
\end{array}\right.
$$

If the width and the height of rectangular cross section is denoted by $w$, and $h$, the maximum stress is

$$
\sigma_{\max }= \pm \frac{3}{2 w h^{2}}(4 F b-P a)-\frac{F}{w h}
$$

where “+” and “-” denote top stress and bottom stress of middle cross section of flexible arm.

\section{Thrust equation of piston in expanding brake}

Displacement and rotation angle of rigidity link can approximate the deformation of flexible department in pseudo-rigid-body model. Thus, rigid body analysis theory can be applied to compliant mechanism. For every flexible arm of expanding brake, pseudo-rigid-body model can be regarded as the combination of joints of torsion spring by rigid rod, which is pictured in Figure 3.

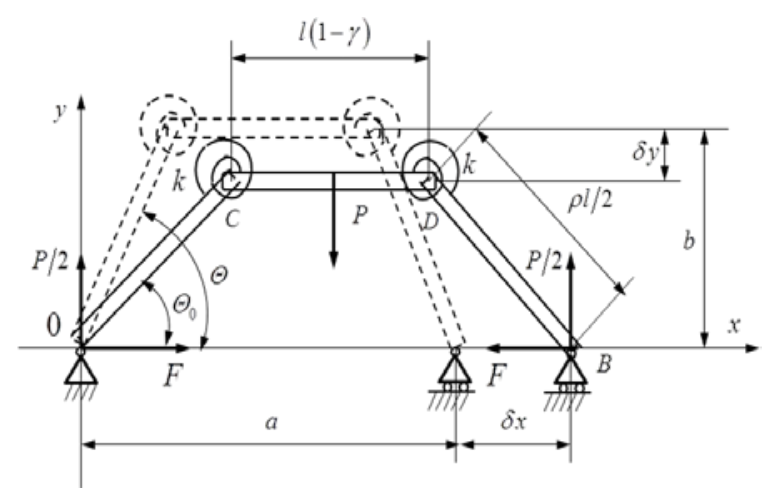

Figure 3 Rigid body model of armed centralizer

Flexible arm can be simulated by $0 C, C D$ and $B D$ segments, where lengths of both $0 C$ and $B D$ are $\rho l / 2$, both of them are bilateral symmetry about action line of expanding force $P$, and $\rho$ is length coefficient. Characteristic pivot is added to between point $C$ and $D$, whose rigidity stiffness coefficient is $K$. It will be used to characterize counter force when flexible arm bending. The distance between Feature hinge and straight rod point is $\gamma l / 2$, where $\gamma$ is the characteristic radius factor of initial horizontal states of both $0 C$ and $B D$. $C D$ will has translational motion not twist motion, while flexible arm are deforming. $O C$ and $B D$ will turn round center of 
gyration about point $\mathrm{C}$ and $\mathrm{D} .0 C$ and $B D$ have initial angle $\Theta_{0}$ in horizontal direction, and the angle will become $\Theta$ after both rods moving.

Let $k_{0}=\frac{l}{R_{0}}$. The stiffness coefficient $k_{\Theta}$, characteristic radius factor $\gamma$, and spring constant $k$ of feature hinge can be described as ${ }^{[5]}$

$$
\begin{aligned}
& k_{\Theta}=2.568-0.028 k_{0}+0.137 k_{0}^{2} \quad 0.5 \leq k_{0} \leq 1.5 \\
& \gamma= \begin{cases}0.8063-0.0265 k_{0} & 0.500 \leq k_{0} \leq 0.595 \\
0.8005-0.0173 k_{0} & 0.595 \leq k_{0} \leq 1.500\end{cases} \\
& k=2 \rho k_{\Theta} \frac{E I}{l}
\end{aligned}
$$

Torsion torque of feature hinge is

$$
T=k\left(\Theta-\Theta_{0}\right)
$$

According to geometry relationship in Figure 3, we can get

$$
\left\{\begin{array}{c}
b=\frac{\rho l}{2} \sin \Theta \\
a=\rho l \cos \Theta+l(1-\gamma)=2\left(2 R b-b^{2}\right)^{\frac{1}{2}} \\
\rho l=\left\{\left(2 b_{0}\right)^{2}+\left[a_{0}-l(1-\gamma)\right]^{2}\right\}^{1 / 2} \\
\Theta_{0}=\arctan \frac{2 b_{0}}{a_{0}-l(1-\gamma)}
\end{array}\right.
$$

By virtual power principle, when flexible arm is in balance, we can get

$$
F=\frac{P}{2} \cot \Theta+\frac{2 k_{\Theta} E\left(\Theta-\Theta_{0}\right) h^{3} w}{6 l^{2} \sin \Theta}
$$

\section{Optimal design of flexible arm}

\subsection{Determination of design variables}

Thrust force should be kept minimum in case of satisfying expanding force while structure designing, which is also the objective of structural optimization.

When designing structure, the amplification degree of pipe diameter should be kept on $\lambda= \pm 10 \%$ because of pipe has local reduced section, dislocation and diameter expansion. If amplification degree of pipe diameter is $-10 \%$, flexible arm is on initial state and expanding force is zero. If amplification degree of pipe diameter is zero, flexible arm is on working state and expanding force is $P$. Pipe diameter $D$ and bearing joint diameter $d$ are always constant. $k_{\Theta}$ and $\Theta$ are often expressed by $l$ and $R$. When expanding force $P$ is constant, we can get the optimal $F$ by combining $l, R, h, w$. So, design variables can be expressed

$$
\mathbf{X}=\left[x_{1}, x_{2}, x_{3}, x_{4}\right]^{\mathrm{T}}=[l, R, h, w]^{\mathrm{T}}
$$

\subsection{Determining objective function}

$$
f(X)=\operatorname{Min}(F)=\frac{P}{2} \cot \Theta+\frac{2 k_{\Theta} E\left(\Theta-\Theta_{0}\right) x_{3}^{3} x_{4}}{6 x_{1}^{2} \sin \Theta},
$$

where

$$
b=x_{2}\left(1-\cos \frac{x_{1}}{2 x_{2}}\right)=\frac{1}{2}(D-d), b_{0}=\frac{1}{2}(0.9 D-d), a_{0}=2 x_{2} \sin \frac{x_{1}}{2 x_{2}}=2\left(2 x_{2} b_{0}-b_{0}^{2}\right)^{\frac{1}{2}},
$$




$$
\begin{aligned}
& k_{\Theta}=2.568-0.028 \frac{x_{1}}{x_{2}}+0.137\left(\frac{x_{1}}{x_{2}}\right)^{2}, 0.5 \leq \frac{x_{1}}{x_{2}} \leq 1.5, \rho x_{1}=\left\{\left(2 b_{0}\right)^{2}+\left[a_{0}-x_{1}(1-\gamma)\right]^{2}\right\}^{1 / 2}, \\
& \Theta=\arcsin \frac{2 b}{\rho x_{1}}, \quad \gamma=\left\{\begin{array}{ll}
0.8063-0.0265 \frac{x_{1}}{x_{2}} & 0.500 \leq \frac{x_{1}}{x_{2}} \leq 0.595 \\
0.8005-0.0173 \frac{x_{1}}{x_{2}} & 0.595 \leq \frac{x_{1}}{x_{2}} \leq 1.500
\end{array} .\right.
\end{aligned}
$$

\subsection{Constraints}

\subsubsection{Geometry constraints}

$$
\left\{\begin{array}{l}
x_{2}>0 \\
x_{1}-D>0 \\
2 x_{2}\left[1-\cos \left(x_{1} / 2 x_{2}\right)\right]+d \leq D
\end{array}\right.
$$

3.3.2 Conditional constraints

(1) Preconditions of both equation (3) and (4) are

$$
\left\{\begin{array}{l}
x_{1}-1.5 x_{2} \leq 0 \\
x_{1}-0.5 x_{2} \geq 0
\end{array}\right.
$$

(2) The force can be regarded as static load because robot moves in low velocity. Flexible permitted stress can be chosen as $75 \%$ of tensile strength, that is,

$$
\sigma_{1,2 \max }= \pm \frac{3}{2 x_{3}^{2} x_{4}}\left[4 F b-P x_{1}(\rho \cos \Theta+1-\gamma)\right]-\frac{F}{x_{3} x_{4}}
$$

\section{Numerical example}

Let pipe diameter $D=150 \mathrm{~mm}$, bearing joint diameter $d=70 \mathrm{~mm}$, flexible arm material be chosen 60Si2MnA, whose Young's modulus E=198GPa, tensile strength $[\sigma]_{b}=1.372 \mathrm{GPa}$, Shear modulus $G=79.84 \mathrm{GPa}$, and Poisson's ratio $\mu=0.29$. When pipe diameter amplification degree $\lambda=0$, expanding force $P=1000 \mathrm{~N}$. After optimization, flexible arm length $l$, radius of curvature $R$, cross section height $h$, width $w$ are calculated as listed in the following Table 1 .

Table 1 Structure parameters of flexible arm unit: $\mathrm{mm}$

\begin{tabular}{cccc}
$l$ & $R$ & $h$ & $w$ \\
\hline 360 & 400 & 7 & 18
\end{tabular}

Experiments shows that mechanism model is correct.

\section{Conclusions}

(A) Pseudo-rigid-body model is adopted, where the nonlinear geometry deformation analysis of flexible can be solved by rigid body theory. Virtual power principle is used to establish static mechanism model for armed centralizer. And the relationship between structure parameters and deformation is induced, which can be optimized. From experimental data, maximum stress will decrease, and thrust force will increase with increasing the cross section diameter and $l 、 R 、 h$ fixed. When cross section size $h$ and $w$ are fixed, piston rod thrust force will decrease with radius of curvature increasing. Structure parameters are chosen optimally for expanding brake of pipe robot with diameter $150 \mathrm{~mm}$.

(B) When determining the relationship between stress and deformation of flexible arm, Calculus operation of nonlinear spring function should be avoided. This paper proposed a simple method for modeling and calculation, which is suitable for low accuracy requirements. Experiments shows that mechanism model is correct. The new method can provide a simple solution for nonlinear structure with large deformation, especially for widely used bow spring casing and automobile suspension 
spring design.

\section{References}

[1] Shi Hongyan. Design and simulation of nonlinear bow spring[J]. Shan Industrial Technology, 2014, (9):7-8.

[2] Ai Chi, Liu Guoyong, Feng Fuping. Impact of Elastic limiting device deformation on middle degree of casing[J]. West-china Exploration Engineering, 2010, 22(2):88-91.

[3] Zhang Haishan, Zhang Kaimin, Gong Jize, et al. Study on influence of different types of centralizer on running casing friction in horizontal well [J]. Drilling \& production technology, 2014, (3):22-25.

[4] HETRIK J A, KOTA S. An Energy Formulation for Parametric Size and Shape Optimization of Compliant Mechanisms[J]. Transaction of the ASME. Journal of Mechanisms in Design, 1999, 121(3): 229-233.

[5] Larry L. Howell. Compliant Mechanisms[M]. Beijing: High Education Press, 2007: 310

[6] Xu Hao. Mechanical design manual(Vol. 4)[M] . Beijing: Mechanical Industrial Press, 1992: $30-15$ 